\title{
Applications of Push Technology in Mobile Social Network Development
}

\author{
Suduo $\mathrm{Li}^{\mathrm{a}}$, Kaiying Deng ${ }^{\mathrm{b}}$, Jingwei Deng $^{\mathrm{c}}$, Yingxing $\mathrm{Li}^{\mathrm{d}}$ \\ College of Mathematics \& Computer Science,North-West University for Nationalities, Lanzhou \\ 730124, P.R.China \\ a435752897@qq.com, b470601995@qq.com, '494521241 @qq.com, diyx555@126.com
}

Keywords: information push technology; mobile social network; mobile application development

\begin{abstract}
With the rapid popularization of smart devices, the traditional social network based on PC is gradually transferred to the mobile Internet. For the problems of mobile devices such as small screen, low power, bad browse experience, etc. using information push technology to publish and access real-time information . This paper introduced the information push technology principle and implementation method. Connecting with the concrete project instance in mobile social development, discussed the configuration, main steps and key technologies to implement information push system. Project operation has obtained a good user experience, shows that the proposed scheme in mobile social development is simple, practical and efficient.
\end{abstract}

\section{Introduction}

In recent years, with the rapid popularization of smart phones, social applications based on instant messaging (also known as "mobile social network") have made full use of the advantages of smart mobile terminal. It greatly satisfied the user's demand for daily communication, and become one of the most favorite applications of users [1].From Facebook, Twitter, YouTube, FourSquare, to Renren, microblog, Youku, Jiepang, from the previous short messages to more and more WeChat blessing, mobile social has gradually become people's indispensable life style. Social networking media is gradually shifting from the traditional PC web to mobile App. However some of the limitations of mobile devices such as small capacity, low power, slow speeds and so on make its application and development very different from traditional PC. On the one hand, it must try to save electricity and traffic as far as possible, on the other hand, it should ensure the data arrive to the client immediately. Information push technology is a powerful tool to solve the contradiction.

\section{Information Push Technology}

Pull and Push. In web applications, there are mainly two ways for a customer to get server data: Pull and Push [2].The difference is that pull is the client requests information actively, while push is the server sends information to client actively. In traditional PC Internet, pull dominate the technology, user will open or refresh a page in need of information so as to get the data from the server. Its advantage is that the information is the latest of the requested time, and also is exactly the user wanted. So the reliability and accuracy of information is very high. The disadvantage is that information timeliness is poorer, when the server data has been updated while the user doesn't refresh the page, the new data will not be perceived by the user. Meanwhile refresh the page also can produce unnecessary network traffic.

Push is a web development technology based on client/server mechanism, which server takes the initiative to send information to clients. Usually the pushed information is user reserved in advance, such as remind new email, prompt new received messages in instant messaging, notify software update to the user timely. By automatically transmit information to users, Push technology reduced user's time for online search, even if the user doesn't start the application he can also receive messages from the application. It search and filter information according to the user's interest, and push it to the user regularly, help user to discover valuable information efficiently. 
Compared with the traditional PCs way to access the Internet, mobile devices are limited in operation convenience and the browser's user experience, and more sensitive to traffic and power consumption, so they have a stronger demand for push. Information push technology gradually used by more and more mobile application developers.

The Technical Principles of Push. In the mobile application development, usually there are three push technologies[3]:

1) Polling mode: This way is to let the client regularly query data on the server. Some applications like email, news and weather that is less demanding for real-time, the simplest and naturally way is that the client periodically connect to the server automatically to execute queries and read data. There are many applications using this way, such as some consulting widget on the Android platform, Shangmail which leading professional mobile email services, etc. This way is not actually push technology, it just achieved push effect from the angle of user experience.

2) Client keeping long IP connection: This way realizes push technology through the client software and server to maintain a persistent TCP/IP connection. The key problem of push to a client IP is that the IP address of the terminal remains relatively stable, and always running the client software and listening for specific socket port, so as to realize the quasi real-time delivery of information [4]. But there are some technical difficulties to keep a long IP connection under mobile wireless network connection, because in mobile communication, long idle connections will be disconnected by network access equipment to save network resources. In addition, mobile terminals are generally use Intranet IP, translating to the public IP need to rely on the gateway NAT service. In order not to disable NAT table, application needs to regularly send "heartbeat" to refresh the NAT table item and avoid to be eliminated. Relative to mobile phone, this way have a larger network traffic and power consumption. Meanwhile the client software needs to be running all the time.

3) WAP push: WAP push system framework includes three functional parts: PI (Push Initiator), PPG (Push Proxy Gateway) and Client PC (Push Client). PI exists in the Internet and PC is in the field of WAP, they are different in the communication protocol, and need to establish a protocol converter that is PPG between them. PPG communicate with PI through PAP (Push Access Protocol), complete data transmission task by Push OTA protocol (push over - the - Air). One of the most common applications of WAP Push is to promote wireless value-added applications, that is send a WAP site or business links through short messages to mobile phones, so the user can access to the business directly via shaort messages listed in the links.

Implementation of Push Technology. The realization of the push technology is started earlier in foreign countries. Both Apple and Google have their own push technologies, such as APNs (Apple's Push Notification service), C2DM (Android Cloud to Device Messaging), etc. [5]. There are also some companies specialized in recommendation, such as Urban Airship. But due to reasons like servers are in foreign countries, it cann't guarantee the information pushed smoothly. These services is difficult to apply in our country.

Push service appeared in China only in recent years. Although some primary and middle developers can rewrite some of the open source code to organize their own push system, but the process is very difficult, and the effect is not well. After all, such technical problems like keeping long connection and processing high concurrent users is not afforded easily by small companies. By contrast, with the aid of the third party platform to realize push effects became a better choice of many primary and middle developers. At present some well-known third-party push platforms in domestic mainly include Yunba, Baidu, Hudee, JPush, Getui, etc.

Using a third-party push platform, developer only need to register an account to push provider, download their push SDK, integrated into his own project, then the developer can program with the interfaces and services provided by push SDK, implement his own push information. The push system includes three roles: the push server, the application server, the application client. Message send by client arrived at application server first, then was encapsulated into a piece of standard push request information transmitted to push server, because push server maintains a long connection to registered application client, so the message can be instantaneously pushed to target users. The principle is shown in figure 1 : 


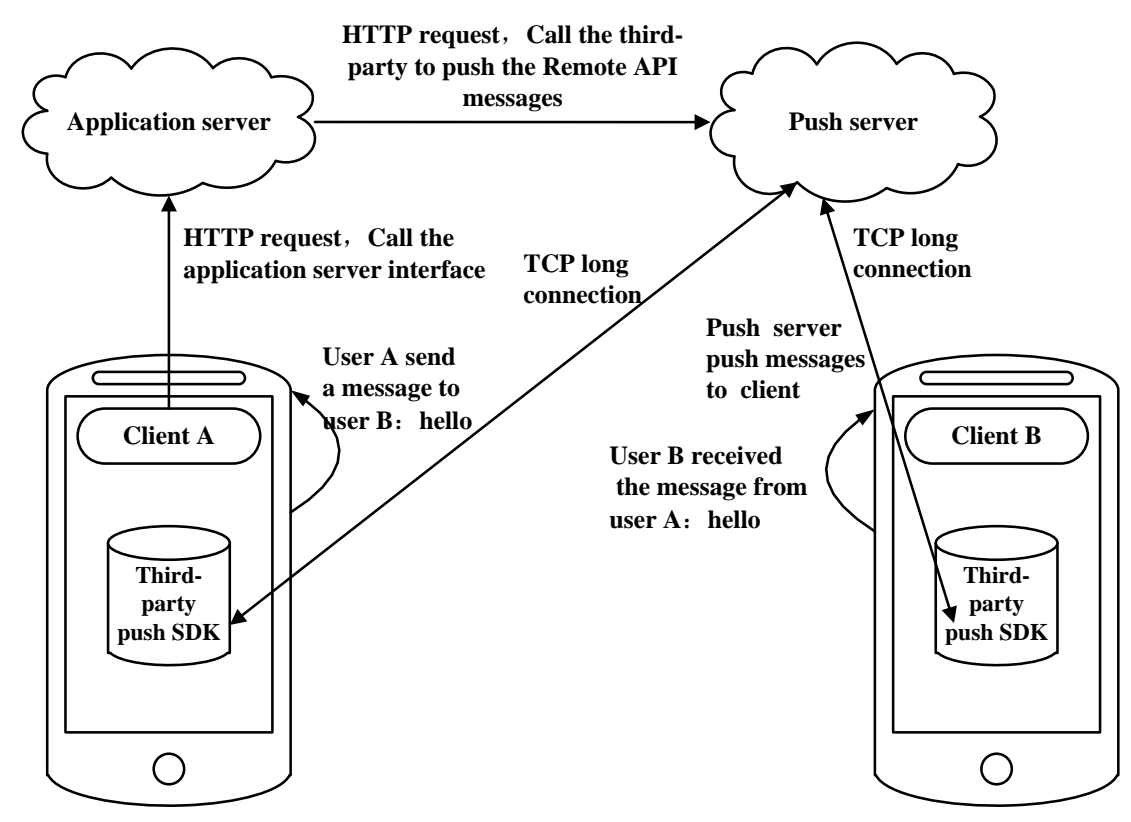

Fig. 1 The principle of third-party push implementation

\section{Example of Push Information Application_— “Cirsaid” Project}

Project Survey. "Cirsaid" is a mobile social applications developed by three students of our Northwest University for Nationalities in software engineering. Compared with some mature social software, such as Momo (location-based social network), Baihe (dating social network), WeChat (acquaintances-based social network), the main characteristic of cirsaid is based on interests, registered users can join a social circle or create a social circle according to their interests. Releasing a piece of information can synchronize to the publisher's all circles. Receiver can filter information according to his like such as only focus on the things of girls of a circle. The project also gave some protection for privacy, it only shows the number of friends and comments, the content and writer of comments are not shown to others, only the two sides of comment can see the detailed information. Besides the project integrates a variety of third-party services, implements many functions such as the validation via SMS, the big data statistics and analysis, location-based services, third-party login, and so on. It formed a practical, powerful mobile phone software that meets people's social demand.

The scene Involved push technology in the project mainly is comments, thumb up and invite friends. It recognize the target of push relying on alias or registration_id, belonging to a single point of push.

Implementation by JPush. This project adopts the JPush as a third party of push platform. It is the most professional third-party mobile information push service provider in domestic. It has provided for thousands of companies and developers stable mobile information push scheme with milliseconds, covered with billions of Android and iPhone terminal. Jpush now support android, iOS and Windows Phone three mobile platforms, even free users can also enjoy the basic push service, basic data statistic and report forms, unlimited number of messages and users, and all-round technical support. Some other advanced application such as rich media push and VIP high-speed push channel will charge a fee.

JPush web site provides the SDK download links, including SDK used in iOS, Android, WinPhone and server-side SDK. If a message is sent by developer, such as notify there is a software update, then you just need to download the client SDK. Developers can directly login background management interface and input message contents, select target of pushed information. This completes the work of push. If the message is sent by a user and push to other users, you would also need to download server-side SDK so that to deal with the message. 
According to the development environment and project requirements they choose the Android SDK and server-side SDK.The Android SDK is used to configure the client running environment of push inforation, its role is as an Android Serivice long running in the background, create and maintain a long connection with JPush servers, keep the clients always online. Detailed introduction of the specific settings is on its website or package of the Android SDK integration guide. The main steps are: firstly imported SDK to your application project, then configure AndroidManifest. XML in the project, add the appropriate permissions and project AppKey in the file.

Invite friends to join the circle for example, the main code is as follows:

private void invitation(final int position, final TextView invi) \{

UserDetailVO userDetail = getItem(position);

List $<$ NameValuePair $>$ valuePairs = new ArrayList $<$ NameValuePair $>$ ();

valuePairs.add(new BasicNameValuePair(PARA_FROM_ID, userId));

valuePairs.add(new BasicNameValuePair(PARA_TO_ID, userDetail.id));

valuePairs.add(new BasicNameValuePair(PARA_CIRCLEID, circleId));

new DoGet(context, REQUEST_URL, valuePairs) \{

@Override

Protected void success(String json, BackMessage $<$ Object $>$ backMessage) \{

if (backMessage.state) \{

invi.setEnabled(false);

invi.setText(TEXTED);

inviation.put(position, true);

\}

\}

\}.execute();

\}

The code is used to select one in the buddy list, click the word "invite" corresponding to the friend to give an invitation. This action builds a valuePairs object that contains three properties: the inviter's ID, the invitee's ID, the ID of the circle that you want your friend to join in. The method DoGet ( )will send the valuePairs to a predefined address REQUEST_URL. After the server processed the request, the friend was set to be have invited.

Server SDK has multiple versions such as Java, PHP, Python, c\#. Our project used Java SDK. After download, import the jar package into the project, and implement the business logic of push in the program.

A piece of push message should state push platform, push target, push content (notification or the message), and other additional options, expressed in JSON format. JPush currently supports Android, iOS, Windows Phone three push platforms. The corresponding key words are: "android", "ios", "winphone". If pushed to all platforms, written as "all". Push target indicates that a message will be pushed to whom. It can be distinguished according to alias, tag, registration_id , broadcast and other means. Push content includes two forms: notification and message. notification will be shown as a"notice" according to certain notification bar style. It may contain different fields in different platform. For example, in the android platform, a notification can contain alert (notice the content), title (notice the title), builder_id (the id of a notification bar style), extras (extension field).Message is used within the application, also called custom message, passthrough message. Its content is not in the notification bar, but need to be shown by the application to deal with. A message typically contains msg_content (message content), title (message header), content_type (the message contents type), extras (additional information), and other fields.

The key step to implement push effect in the program is to build a PushPayload object which encapsulates a push information. PushPayload is a wrapper class to translate the corresponding attributes into json format and sends it to Jpush server. Due to the diversity of push platform, push target and push content, it need to build more PushPayload objects with different parameters, such as:

public PushPayload buildPushObject_all_all_alert(String content) \{

return PushPayload.alertAll(content); 
Is to push a notice with content "content" to all platforms and all users. Another example:

public PushPayload buildPushObject_all_alias_alert(String alias, String content) \{

return PushPayload.newBuilder().setPlatform(Platform.all()).setAudience(Audience.alias(alias)) .setNotification(Notification. alert(content)).build();

\}

This code has alias and content two parameters. It means to push a notice to users whose alias name is "alias" and regardless of platform. Other methods of constructing PushPayload object is similar.

As for the specific components of a notice such as the title and the content, need to assign its values with special statements. For example, when invite friends to join the circle, the code is as follows:

title = String.valueOf(TYPE_INVITATION);

content $=$ messageVO.getFrom_nickName ()$+"$ Invite you to join "+ messageVO.getCirSaidContent();

Other code, such as building the message, send the message, handle exceptions, etc., will not described in this article due to the limit of space.

\section{Project Performance}

"Cirsaid" project started a trial run since June 2015. The server-side is deployed in Aliyun cloud server appeared in recent years. Aliyun's cloud host is based on the famous Feitian cloud computing systems, it is used by Taobao, Tmall etc. as underlying architecture and has excellent performance of high concurrent access. And the hard disk of Aliyun's cloud host is planted on a separate network storage cluster. Each data has three copies. Once write a data, it also writes to different locations in the cluster through cluster switches at the same time. Thus ensuring the high reliability of the data.

At present, the project is still in trial stage. There are more than four hundred registered users to participate in user experience and feedback, mainly used by young people. According to present usage, the basic function designed in the early stage of the project has been implemented. The system runs stable and reliable. But sometimes push operation based on JPush will be a short delay, maybe for a few minutes. This will not affect social applications too much. However occasionally user may not receive the message pushed to him at all. This case is related to user's network status, has instructed on JPush official document. Other aspects of the project is still in a functional expansion and improvement of the system. Later they will enhance the propaganda. It should have a good prospect.

\section{Conclusion}

With the vigorous development of the mobile application developing, the importance of information push technology increasingly highlighted. Pushed by the third party platform, we implement active push information in the "cirsaid" project, and win a good user experience, preliminary shows the superiority of push technology in mobile social network development. However, the push technology involved in this project is just a simple single point push, that is the user actively generates push information and specify to push it to another users. How to integrate artificial intelligence, knowledge discovery technology, Internet and database technology based on the current push technology, thus forming an intelligent information push technology, will be the future development direction of the push technology [6]. 


\section{Acknowledgment}

This work is supported by the Fundamental Research Funds for the Central Universities of China (13920150080); Humanities and Social Sciences Youth Projects of the Ministry of Education of China(12YJCZH027, 13YJCZH029).

\section{References}

[1] Yao Cheng, Lingyun Ying, Sibei Jiao, etc. Research on User Privacy Leakage in Mobile Social Messaging Applications. [J]. Chinese Journal of Computers, 2014 (1) : pp.87-99.

[2] Youjun Yan, Lei Xu, Lin Chen, etc. Research on Web Service Push Techniques Based on Mobile Platform [J]. Journal of Frontiers of Computer Science and Technology, 2012 (7) : pp.602-611.

[3] Changxue Zhang, Wei Zhang, Zhiming Dong. Aspects of Mobile Push Technology [J]. Journal of mobile communications, 2011 (5) : pp.21-27

[4] Yongzhi Yan. HTTP-Based Long-Connect "server push" of Technology Research [D]. Changsha: Hunan University, 2010

[5] Jicheng Wei, Shuqin Li, Lili Yang. Application of Data Push Technology in the Control and Management of Greenhouse Irrigation [J]. Hubei Agricultural Science, 2014 (9) : pp.4441-4444.

[6] Yingqing Liu. Summary of the Information Push Research in China [J]. Journal of Changsha University, 2006 (9) : pp.82-86. 